\title{
Meta
}

Journal des traducteurs

Translators' Journal

\section{Henry, J. (2003) : La traduction des jeux de mots, Paris, Presses de la Sorbonne Nouvelle, 297 p.}

\section{Hélène Buzelin}

Volume 49, numéro 2, juin 2004

URI : https://id.erudit.org/iderudit/009360ar

DOI : https://doi.org/10.7202/009360ar

Aller au sommaire du numéro

Éditeur(s)

Les Presses de l'Université de Montréal

ISSN

0026-0452 (imprimé)

1492-1421 (numérique)

Découvrir la revue

Citer ce compte rendu

Buzelin, H. (2004). Compte rendu de [HENRY, J. (2003) : La traduction des jeux de mots, Paris, Presses de la Sorbonne Nouvelle, 297 p.] Meta, 49(2), 387-391.

https://doi.org/10.7202/009360ar

Ce document est protégé par la loi sur le droit d'auteur. L'utilisation des services d'Érudit (y compris la reproduction) est assujettie à sa politique d'utilisation que vous pouvez consulter en ligne.

https://apropos.erudit.org/fr/usagers/politique-dutilisation/
Cet article est diffusé et préservé par Érudit.

Érudit est un consortium interuniversitaire sans but lucratif composé de l'Université de Montréal, l'Université Laval et l'Université du Québec à Montréal. Il a pour mission la promotion et la valorisation de la recherche. https://www.erudit.org/fr/ 
que dénonce fort justement Gaudin lui-même!) - mais insuffisamment pour un lecteur qui n'a pas lu L'Archéologie du savoir. L'auteur ne s'étend guère non plus sur la praxématique ou la subduction guillaumienne.

Enfin, il faut hélas souligner que l'éditeur - qui a par ailleurs démontré son dévouement à la cause universitaire et son savoir-faire dans cette même collection et dans d'autres - n'a pas ici excellé: s'il a fait en sorte que l'ouvrage comporte deux index, l'un pour les notions et l'autre pour les noms propres, il a par contre laissé de trop nombreuses coquilles, typographiques ou de formulation ${ }^{1}$, un certain nombre de références bibliographiques qui ne renvoient à rien dans la bibliographie (Gaudin 2000e cité page 228; Dahan-Dalmédico pages 236 et 242), et une bibliographie très désorganisée (ouvrages cités deux fois, ordre alphabétique bouleversé, etc.).

En conclusion, l'ouvrage de François Gaudin a peut-être été écrit et réalisé trop vite, et il sera d'un abord difficile pour les débutants. Mais il est important par son sujet, par son originalité, par l'attention qu'il porte aux aspects discursifs, sociolinguistiques et historiques trop souvent négligés de la naissance et de la vie des termes, et il sera dorénavant un must (Monsieur Toubon et sa loi me pardonnent) pour les étudiants de terminologie. Espérons qu'ils ne tireront pas de ses raccourcis et de la densité de son style la conclusion abusive que la terminologie est une chose bien complexe.

HENRi BÉJOINT

Université Lumière Lyon 2, Lyon, France

\section{NOTES}

1. J'en indique ici les références, au cas où il y aurait une réimpression ou une seconde édition : 16, 17, 19, $56,63,64,65,69,84,86,91,122,127,129,141,156,157,159,160,161,165,169,176,178,179,180$, $181,182,187,189,196,201,220,223,240,243$.

Henry, J. (2003) : La traduction des jeux de mots, Paris, Presses de la Sorbonne Nouvelle, $297 \mathrm{p}$.

Cet ouvrage, au titre explicite, explore une question sur laquelle la plupart des théoriciens de la traduction ou de la littérature se sont, un jour ou l'autre, prononcés mais qui, à ma connaissance, n'avait pourtant encore jamais fait l'objet d'une étude aussi approfondie. Ne serait-ce que pour cette raison, le travail de Jacqueline Henry est particulièrement bienvenu et offre une contribution importante aux études en traduction. Réfutant le parti pris théorique d'intraduisibilité des figures de style, et selon une perspective fonctionnaliste ancrée dans la théorie du sens de Lederer et Seleskovitch, l'auteure s'attache à démontrer les mécanismes des jeux de mots, leurs fonctions et effets ainsi que la variété de stratégies qui s'offrent à ceux et celles qui ont pour tâche de les restituer en traduction. L'ouvrage se divise en quatre chapitres. Les deux premiers, d'orientation théorique, délimitent l'objet d'étude et offrent un panorama des recherches qui s'y rattachent. Les deux derniers explorent consécutivement les enjeux que soulève la traduction de deux types de jeux de mots: ceux qui jouent un rôle ponctuel et secondaire dans le texte (chapitre 3 ) et ceux qui sont constitutifs de son système d'écriture (chapitre 4). La réflexion se nourrit des multiples exemples que l'auteure a puisés, entre autres, dans sa propre pratique de traduction des textes de D. R. Hofstadter (Gödel, Escher, Bach, Les Brins d'une Guirlande Eternelle et Vues de l'esprit), de Shepard (L'oil qui pense), de Wolfe (Free Live Free) et de Campanile ainsi que dans diverses traductions de textes classiques, depuis The Bible jusqu'à Finnegans Wake, en passant par Alice's Adventures in Wonderland. Les principales langues à l'étude sont le français, l'anglais et l'italien. 
Le premier chapitre énonce donc le cadre théorique adopté tant du point de vue des jeux de mots que de celui de la traduction. L'auteure y présente et commente les principales tentatives de classification des jeux de mots (Freud, Guiraud, le Goupe Mu, Landheer), relève les limites de chacune et adopte celle de Guiraud, laquelle repose non pas sur des critères morphologiques, mais sur le type d'opération effectué: substitution, enchaînement ou permutation. Les caractéristiques généralement associées au jeu de mots sont ensuite analysées en détail : les fonctions linguistiques, l'humour, la duplicité, la connotation, l'oralité, la connivence avec le destinataire. La discussion révèle, entre autres, les liens entre la traduction des jeux de mots et celle, plus générale, des connotations. L'auteure démontre les limites d'une conception qui ferait de ces manifestations textuelles des phénomènes de surface, donc seconds. Elle insiste au contraire sur la nécessité d'interpréter et de rendre les fonctions textuelles (didactique, ludique, mnémotechnique, subversive, poétique, etc.) du jeu de mots et la réaction qu'il cherche à susciter. Cette perspective débouche sur une nouvelle classification relative au poids des jeux en question dans le texte à traduire, classification dont la pertinence traductologique sera démontrée aux chapitres trois et quatre. On y distingue les jeux de mots ponctuels, ceux qui font partie du système d'écriture du texte, et ceux qui en constituent le fondement même. Si les frontières ne sont pas étanches, ces trois cas de figure soulèvent, selon l'auteure, des questions différentes et ne requièrent pas tout à fait la même approche traductionnelle. Le chapitre se conclut sur un rappel des fondements de la théorie de l'interprétation de Lederer et Seleskovitch dans laquelle s'inscrit cette étude.

Le deuxième chapitre est une critique, nuancée mais solide, de la "prétendue intraduisibilité» des jeux de mots, et plus généralement de la forme signifiante. L’auteure rappelle les positions pour le moins défaitistes qu'entretiennent en la matière les traductologues, linguistes ou critiques littéraires tels que George Mounin, Eugene Nida, Catford, Claude Hagège, Hans Teichmann, Marina Yaguello et Jean-Louis Curtis. Au-delà des perspectives particulières dans lesquelles travaillent - ou travaillaient - ces chercheurs (la traduction automatique pour l'auteur de A Linguistic Theory of Translation, la traduction biblique dans une visée de prosélytisme pour Nida et Taber, par exemple), ce défaitisme tient, selon elle, tantôt à une méconnaissance de la nature des langues et/ou des jeux de mots, tantôt à une conception erronée de la traduction. Ainsi, la thèse selon laquelle les jeux de mots seraient des accidents de langue "pêche par généralisation et néglige le fait que les langues n’ont pas évolué de façon totalement isolée les unes des autres, ne serait-ce qu'en raison, parfois, de leurs liens de "filiation»» (p. 70). De fait, les chapitres suivants offriront de nombreux exemples attestant la façon dont le traducteur peut parfois tirer partie des liens entre des cultures et lexiques a priori distincts. Cette thèse repose également, toujours selon l'auteure, sur une définition réductrice du jeu de mots qui se limite «aux jeux sur les plurivalences (calembours) en oubliant les autres catégories» (p. 72) et qui envisage ces derniers sous angle exclusivement formel, accordant plus d'importance aux signifiants en eux-mêmes (au matériau sonore ou visuel exploité) qu'aux fonctions et effets dont ils sont investis; bref, une définition qui fait fi du rapport entre le jeu de mots et le texte (et à plus forte raison le contexte) dans lequel il s'inscrit. Enfin, cet a priori d'intraduisibilité dénoterait la persistance d'une définition de la traduction comme simple recherche d'équivalents (dans le cas de Nida, Taber et Catford), voire de calque (dans celui de Curtis). Ainsi, en analysant les discours des spécialistes, et en critiquant plus particulièrement les théories traductologiques d'inspiration linguistique, l'auteure reflète-t-elle les contradictions internes aux théories en question. Ces dernières, qui admettent par ailleurs la complexité du processus de traduction, resteraient prisonnières de la dichotomie fond/forme et continueraient de véhiculer l'idée voire l'idéal - de la traduction-transcodage ou de la traduction-exégèse. Selon cette conception dualiste, la ré-énonciation, dans une autre langue, des jeux de mots, tout comme celle des textes poétiques, relèverait non pas de la traduction, mais bien plutôt de l'adaptation. Telle 
était d'ailleurs l'opinion de Roman Jakobson. Ce constat, qu'elle réfute, conduit l'auteure vers une réflexion plus générale sur les liens et différences entre la poésie et les jeux de mots. Si l'une et les autres ont en commun de s'éloigner, intentionnellement, d'une utilisation purement référentielle de la langue et de briser l'arbitraire du signe, la première met en œuvre une plus grande variété de figures. La différence en est donc une d'échelle. La réflexion débouche sur une tentative de «redéfinition du sens en traduction» (p. 106). Aux conceptions dualistes critiquées précédemment, l'auteure propose de substituer une vision fondée, d'une part, sur le constat de l'universalité des jeux de mots et de leurs fonctions et, d'autre part, sur la «théorie du sens». Ainsi, le traducteur doit-il savoir avant tout reconnaître «le sens de la forme», la visée globale du texte à traduire, le projet de l'auteur, autrement dit son vouloir-dire.

Ce n'est qu'une fois qu'il a compris la nature du projet réalisé à travers l'œuvre à traduire que le traducteur peut définir son propre projet analogue et déterminer comment il pourra faire «la même chose» [...]. Dans l'univers d'accueil du texte second, c'est-à-dire à son époque, dans sa culture, avec les moyens et les conventions de sa langue, le traducteur s'efforcera alors de recréer l'original, de produire une ouvre 'ressemblante'. Il ne peut viser l'identité ou la copie, au sens où l'on peut reproduire une copie d'un tableau ou d'une statue, mais plutôt une œuvre équivalente, analogue, qui, dans ses conditions de réception, a une fonction et un effet aussi proches que possible de ceux de l'original. (p. 108)

En ce sens, seuls les jeux de mots dont la motivation demeure obscure, tel celui de Max Jacob dans Le cabinet noir: "Tout enfant, je fus élevé dans un chambre à nourrice dessiné par Steinlen, c'est pourquoi j'adore les chats (jeu de mots intraduisible)...» (p. 110), seraient intraduisibles. Au terme de ce chapitre riche, instructif et parfois décapant, on peut juste regretter que l'auteure n'ait pas pris autant de recul avec «la théorie du sens» - qui a pourtant fait l'objet de critiques depuis ses premières formulations - qu'avec celle, entre autres, de Nida et de Taber dont les limites ont été souvent relevées au cours des vingt dernières années. Cette absence surprend d'autant plus qu'au vu de certains passages, tels que celui figurant ci-dessus, on se demande à quel point, en pratique, son approche traductionnelle est véritablement différente de celle que recommandaient les auteurs de The Theory and Practice of Translation.

Après avoir exposé les fondements théoriques de son étude, l'auteure aborde, dans le troisième chapitre, les questions touchant plus particulièrement «la traduction des jeux de mots ponctuels », ceux qui sont dotés d'une relative autonomie et ont un impact local dans le texte de départ. Le chapitre analyse tout à tour les paramètres d'ordre linguistique, textuel, contextuel et cognitif susceptibles de conditionner l'interprétation puis la traduction de ces jeux de mots. Y est ainsi évoquée, de multiples exemples à l'appui, l'influence des caractéristiques propres aux langues de départ et d'arrivée - toutes les langues ne se prêtent pas également aux mêmes types de jeux - et aux traditions littéraires qui s'y rattachent, caractéristiques dont le traducteur, "sous peine de produire des résultats forcés» (p. 134), doit savoir tenir compte. Tout aussi importante est l'identification des fonctions du jeu de mots au sein du texte à traduire. Les principales, dont chacune fait l'objet d'un examen et d'une ou plusieurs illustrations, sont l'accroche (retenir l'attention), l'amusement (faire rire ou sourire le lecteur), la caractérisation (indiquer la personnalité d'un actant), la virtuosité (susciter l'admiration du lecteur), le reflet du sujet représenté (lorsque le jeu de mots illustre le contenu du propos). Le choix entre plusieurs stratégies de traduction sera aussi, dans une large mesure, fonction du contexte dans lequel s'inscrit le jeu de mots, que ce soit le contexte verbal immédiat ou la trame narrative.

Le traducteur peut en effet trouver des moyens de résoudre son problème en examinant le tissu verbal ou cognitif proche du jeu de mots à transposer ou en s'appuyant sur des éléments à un niveau plus macroscopique dans le texte à traduire (p. 160). 
Enfin, le quatrième et dernier paramètre, de nature très différente des précédents, touche au «bagage cognitif» du traducteur. D'après les illustrations fournies, ce critère renvoie aux connaissances culturelles et pragmatiques relatives au contexte-cible. Ces dernières offrent une «riche source de solutions traductionnelles» (p. 173) que le traducteur doit toutefois savoir mobiliser, à bon escient: " pour que les jeux de mots ainsi trouvés puissent être satisfaisants, cette exploitation doit être bien maîtrisée et ramenée au cadre du texte et de ses destinataire» (p. 173). On en revient donc à la fonction et aux effets recherchés, ultime déterminant des choix de traduction, du moins selon l'auteure. Les nombreux exemples étudiés tout au long du chapitre font ressortir l'étendue de la marge de manœuvre du traducteur, la diversité des stratégies possibles. Ces dernières sont finalement répertoriées dans une typologie où l'auteure distingue 1) les «traductions isomorphes» (jeu de mots identique dans les deux textes) relativement rares, 2) les «traductions homomorphes» (jeu de mots différent mais reposant sur un même procédé) souhaitables et souvent possibles, 3) les traductions «hétéromorphes» (jeu de mots reposant sur un procédé différent) parfois nécessaires lorsque la stratégie précédente est impossible, 4) la traduction libre (création d'un jeu de mots absent dans l'original, ou inversement)... qui ne relève plus tout à fait de la traduction.

Le dernier chapitre examine enfin «les problèmes spécifiques posés par la traduction de textes construits autour de jeux de mots [afin de] déterminer avec une certaine précision la nature de l'opération réalisée lors du transfert de ces textes d'une langue à une autre» (p. 193). La réflexion se fonde en grande partie sur plusieurs versions françaises de deux nouvelles de l'écrivain italien Achille Campanile tirées du recueil Manuale di conversazione (1973) : «La quercia del Tasso» et «La rivolta delle sette», nouvelles dont la trame narrative est, sinon insignifiante, du moins relativement mince, et dont les jeux de mots constituent la principale raison d'être. Après avoir analysé l'original et ses propres traductions, Jacqueline Henry explique les liens mais surtout les différences entre les enjeux que posent la traduction de ces nouvelles dont la densité en jeux de mots est particulièrement élevée, celle des textes de Carroll ou de Shepard - qui tirent une partie mais non l'essentiel de leur pertinence de ces jeux verbaux -, et celle de textes de type plus pragmatique où les jeux de mots interviennent de façon locale et restreinte. Selon l'auteure, les premiers requièrent une approche macrotextuelle visant à restituer l'effet global, la tonalité et le tissu de jeux verbaux de l'original, quitte à modifier la trame narrative qui vient les légitimer.

[...] tout comme les auteurs de ces écrits sont plus libres, parce qu'ils n'opèrent pas dans un seul domaine notionnel, le traducteur est lui aussi plus libre, une fois qu'il a dégagé la «machine» du texte parce qu'il opère clairement dans le domaine de la re-création, ce qui lui demande de mettre en œuvre sa science de la traduction, mais aussi ses qualités d'inventivité, d'originalité et d'écriture, bref, son art de la traduction (p. 219).

En somme, ces textes ne sont pas plus intraduisibles que les autres; par contre, il convient de déterminer le type d'opération dont ils font l'objet. L'approche "macrotextuelle » préconisée par l'auteure conduit donc à une réflexion sur la nature du processus en œuvre, et plus particulièrement sur la notion d'adaptation généralement invoquée dès lors que la traduction dépasse un certain seuil de liberté. La suite du chapitre retrace l'origine de cette notion, sa polysémie et plus particulièrement les connotations souvent négatives, parfois positives, qui y sont associées. L'auteure commente en détail la thèse de Georges Bastin ${ }^{1}$ et prend position: reconnaissant d'une part la pertinence de la distinction qu'il établit entre adaptation tactique (locale) et stratégique (globale), elle réfute, de l'autre, l'idée selon laquelle la première serait facultative. À la suite d'Yves Gambier², Jacqueline Henry nie la possibilité d'établir une ligne de démarcation entre cette notion et celle de traduction et propose d' «en finir avec l'opposition traduction-adaptation» (p. 242). D'autres notions, telles que celles analysées par Genette dans Palimpsestes - forgerie, pastiche, parodie ou transposition, etc. permettraient selon elle d'éviter ce dualisme (et les valeurs dont il se charge) et de décrire avec plus de précision le type d'opération à l'œuvre. 
La traduction des jeux de mots est un ouvrage extrêmement riche, instructif et de lecture très agréable. Outre sa contribution immédiate à une question souvent évoquée par les théoriciens de la traduction, mais rarement traitée en profondeur, cette étude a entre autres le mérite de relever les contradictions qui entourent certaines théories contemporaines de la traduction. Ce faisant, elle pose aussi des questions beaucoup plus vastes relatives à la nature même du processus de traduction, aux liens entre traduction, adaptation et recréation, au rôle du sujet traduisant. L'intérêt de l'ouvrage réside également dans la diversité et l'étendue des exemples que l'on y trouve. Bien qu'elle ne brise pas complètement l'opposition «littéraire» / «pragmatique» - qui prend ici la forme d'un continuum: les jeux de mots étudiés dans le dernier chapitre apparaissant uniquement dans des textes littéraires - Jacqueline Henry illustre sa réflexion en mobilisant, en juxtaposant parfois même, des exemples puisés dans tous les genres: des textes sacrés à la littérature jeunesse, en passant par la fiche technique, la publicité, la poésie, voire le slogan politique. Enfin, la principale qualité de cette étude tient, à mon sens, à l'équilibre qu'elle parvient à instaurer entre les perspectives théorique et pratique. Bien que l'ensemble se divise en deux parties, la première étant plus théorique, la seconde plus pragmatique, chaque chapitre est construit de façon à créer un dialogue entre ces deux points de vue. Jacqueline Henry n'hésite pas à mettre à profit son expérience de traductrice pour reconsidérer certains préjugés théoriques et poser de nouvelles questions; elle parvient tout aussi bien à rattacher des questions a priori pragmatiques à des débats plus vastes. Ce mouvement incessant entre théorie et pratique crée une dynamique qui rend la lecture du texte particulièrement stimulante. Cet ouvrage très clair, accessible (un glossaire des concepts clés de l'étude est fourni en annexe) et d'orientation assez didactique pourrait constituer un excellent texte de référence dans les cours de traduction littéraire ou d'adaptation. Il intéressera également tous ceux, professionnels et universitaires -traductologues, linguistes ou critiques littéraires -, qui pratiquent la traduction ou s'y intéressent.

HÉLÈne BUZELIN

Université de Montréal, Montréal, Canada

\section{NOTES}

1. La notion d'adaptation en traduction - Étude de l'adaptation ponctuelle et globale dans la version espagnole de "L'analyse du discours comme méthode de traduction » de J. Delisle. Thèse de doctorat de $3^{\mathrm{e}}$ cycle, Paris III-ESIT, 1990.

2. Yves Gambier, «Adaptation: une ambiguïté à interroger », Meta 29-4, 1992, p. 421-425.

Van Hoof, H. (2003): Les mots de la chose. Dictionnaire de l'érotisme (français et anglais), Paris, Pauvert, $450 \mathrm{p}$.

Même si le terme érotisme ne date que de 1794, selon le Petit Robert, la chose est aussi vieille que l'espèce humaine! Comme le rappelle avec justesse l'auteur dans son Introduction: «Ce qui intéresse l'expression érotique, ce n'est point la réalité physique du sexe, mais la signification attribuée au sexe dans l'imagination des peuples. Et l'inconscient collectif trouve là un domaine où il peut donner libre cours à sa créativité, érotisant jusqu'aux vocables les plus neutres (en français: affaire, chose, faire, etc.; en anglais: affair, do, thing, etc.)» D'où le titre de l'ouvrage! Il suffit de parcourir les rues de n'importe quelle ville pour voir, et même parfois admirer l'exquise subtilité érotique des affiches publicitaires mettant en relief des vêtements ou divers accessoires ou tout simplement des voitures. Les jugements sagaces des publicitaires savent que l'érotisme est «bon vendeur»! Bien sûr, la mince limite entre l'érotisme et la pornographie est parfois franchie. 